\title{
Ein Fall von Störung der Reizleitung im Herzmuskel.
}

Von Dr. James Mackenzie in Burnley (England).

Beim Lesen von Wenckebachs Buch ,die Arhythmie als Aus-

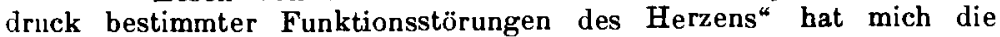
Analyse seiner Pulskurven, wo er die Allorhythmie einer Störung der Reizleitung zuschreibt, besonders interessiert. Die Uebereinstimmung zwischen den von ihm analysierten Pulsunregelmäßjigkeiten und den experimentelleı Herzallorhythmien ist lı̈chst iiberraschend; trotzdem kam anfangs ein Zweifel in mir auf, weil ich bei der Analyse vieler meiner eigenen Pulskırven, welche eine oberflächliche Aehnlichkeit mit den von Wenckebach beschriebenen Formen hatten, in den gleichzeitigen Kurven von arteriellen und venösen Pulsen keine Andeutıng einer Verzögerung der Leitung rom Vorhof zum Ventrikel finden konnte. Umsomehr frente es mich vor kulzem, eine Kranke mit unl'egelmälbigem Pulse zu finden, bei welcher die gleichzeitige Registrierung des Jugular- und des Radialpulses gestattete, die Erklärung Wenckebach $s$ anfs vollständigste zll bestatigen.

Die betreffende Kranke wal eine 24 jährige Frall, primigravida im fünften Monate, welche mich am 1. September 1903 konsultierte. In 1899 war sie wegen Mitralinsuffizienz unter meiner Behandlung. In 1901 machte sie einen $\Lambda$ nfall von rheumatischem Fieber durch, in den letzten achtzehn Monaten aber war sie in guter Gesundheit, nur etwas kurzatmig bei Körperbewegung. Am 10. September hatte sie einen regelmäßigen, frequenten und weichen Puls; es war eine dentliche Pulsation in den tiefen Halsvenen wahinehmbar, welche ich gleichzeitig mit dem Radialpulse registrierte (Fig. 1). Die Herzdämpfung

Fig. 1.

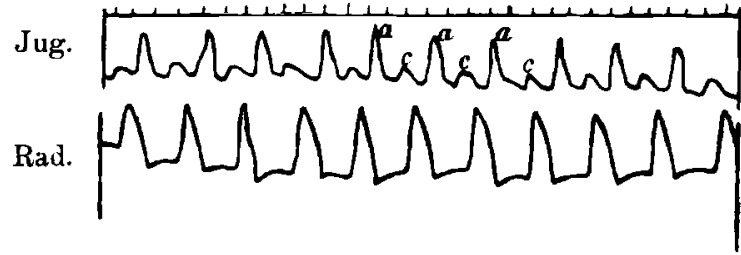

Gleichzeitig registrierte Jugular- nnd Radialpulse. Die Welle $a$ ist eine venöse, durch die Systole des rechten Vorhofs verursachte Welle, $c$ die fortgeleitete Karotiswelle, der Intervall $a-c$ milbt $8 / 10$ Sekunden.

reichte nach links bis außerhalb der Mamillarlinie, der Herzstoß war in del Mamillarlinie im fünften Interkostal raum fühlbar; an der Herzspitze hörte man ein blasendes Gerä̈lsch, welches sich bis in die Axilla fortpflanzte. Die P'atientin blieb in mäßjig gutem Zustande bis zum 4. November, als sie über Schwächegefühl und Kurzatmigkeit klagte. Das Herz schlug regelmäßig, aber frequent (100 per Minute), Oedeme fehlten, aber der U rin war spärlich. Ich verschrieb 10 Minims Tinctura digitalis ( $\mathrm{Br}$. Ph.), dreinnal täglich. Am 8. November war sie nicht besser, der Puls blieb frequent und zeigte dann und wann eine Intermission, wie in Fig. 2. Am 11. November war Patientin

Fig. 2.

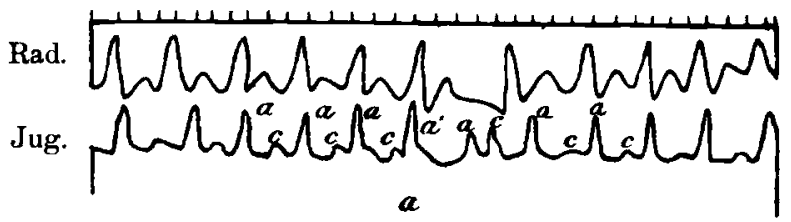

Es erfolgt eine Intermission, nach derselben in $a-c$ kürzer, als vorher.

noch schwächer, die Kurzatmigkeit hatte zugenommen. Der Puls war nun sehr unregelmäßig: eine Zeitlang folgte auf je zwei oder drei Pulse eine Pause (Fig. 3, 4) dann wieder wurde der Puls regelmäBig, aber über 100 pro Minute. Digitalis wurde nicht mehr verabreicht, die Kranke erholte sich dann langsam, bis am 18. November der Puls regelmäßig wurde, mit einer Frequenz von 80 pro Minute. 
Fig. 3.

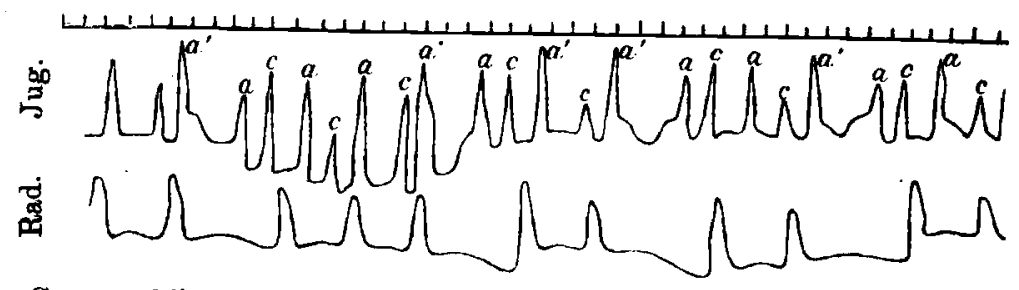

Gruppenbildung des Radialpulses. Nach jeder dritten $a$ fällt $c$ aus Nach diesem Ausfall ist $a-c$ immer kürzer.

Ehe ich zur Analyse der hier abgebildeten Kurven schreite, möchte ich in kurzem mitteilen, wie dieselben gewonnen wurden. Ich lege eine kleine offene Schüssel über die Jugularvene, genau oberhalb des Schlüsselbeins. Diese Schüssel steht durch einen Gummischlauch in Verbindung mit einer Mareyschen Trommel, welche an einen

Fig. 4.

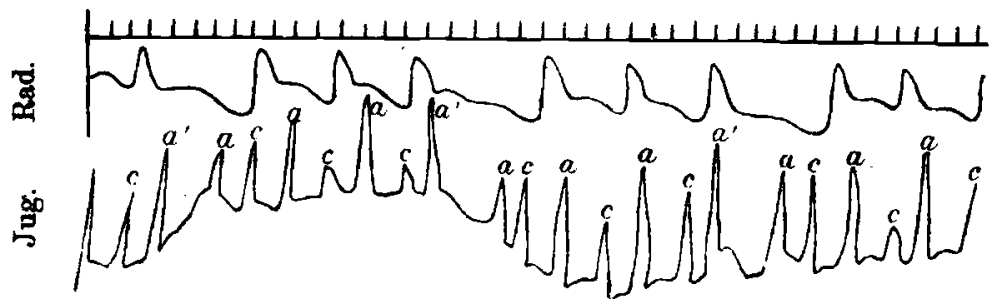

Nach jeder vierten $a$ fällt $c$ aus.

Dudgeonschen Sphygmographen in der Art befestigt ist, daß der Schreibhebel gleichzeitig mit dem vom Radialpuls bewegten Zeiger des Sphygmographen auf das beruBte Papier schreibt. Um die Venenbewegungen richtig zu deuten. braucht man sich nur zu merken, welche Stellung dieselben in der Herzrevolution einnehmen durch Vergleichung mit dem Radialpulse. Mit den Venenbewegungen wird sehr oft auch dis arterielle Karotiswelle registriert, weil der A ufnahmeschlüssel auch die Karotisgegend bedeckt. So findet sich auch in den hier abgebildeten Kurven neben der von der Vorhofstätigkeit verursachten venösen Welle a die von dem arteriellen Karotispulse verursachte kleine Welle c. Für die Beweise für diese Dentung verweise ich auf mein bald auch in deutscher Uebersetzung erscheinendes Buch uber den Puls; hier sei nur darauf hingewiesen, wie c beim Ausfall des Radialpulses ebenfalls ausfällt nnd dadurch schon ihre arterielle Natur kundgibt. Die Zeitschreibung zeigt $1 / \mathrm{s}$ Sekunde an.

Es kommt in diesen Kurven nun darauf an, sich die variable Stellung der arteriellen Welle $\mathrm{c}$ (und des Radialpulses) in ihrer Beziehung zu der von der Vorkammerkontraktion herrührenden Welle a zu markieren. So lange der Puls noch regelmäßig war (Fig. 1), war das Intervall a-c (also Vorkammer-Kammerkontraktion $=\mathrm{As}_{\mathbf{s}}-\mathrm{V}_{\mathrm{s}}$ ) schon größer, als in der Norm. In Fig. 1 ist die Dauer des Intervalls $8 / 10$ Sekunde, in Fig. 2 schon beinahe $1 / 10$ Sekunde. Dabei ist zu bedenken, daß bei unserer Patientin die Frequenz viel zu hoch war. In Fig. 2 fallen eine Radialis- und Karotiswelle aus. Trotzdem fehlt die Vorhofswelle $a^{1}$ nicht; es ist nun interessant, festzustellen, daß auch die durch den Kammersystolenausfall bedingte Pause a-c viel kürzer ist, als sonst. In Fig. 3 erfolgen die Radialpulse in Gruppen, die erste Gruppe enthält drei, die folgenden je zwei Pulse; hier fällt, wie aus dem Venenpulse hervorgeht, nach jeder dritten $A_{s}$ eine $V_{s}$ aus; die $A_{s}$ aber erfolgen durchaus rhythmisch und regelmäßig. Die arteriellen Pulsperioden zeigen nicht nur Pausen, sondern sind auch unter sich ungleich lange; in jeder Gruppe aber (vergleiche Fig. 3 und 4) kehrt diese Ungleichheit in regelmäBiger Weise wieder, die erste Periode nach der Pause ist die längste, die zweite die kürzeste. Bei näherer Betrachtung kommt nun heraus, daB die regelmäBige Vorhofspulsation zu der Kammerallorhythmie führt, durch die das zeitliche Verhältnis zwischen $A_{s}$ und $\nabla_{s}$ gestört ist. Nach jeder Pause ist das Intervall a $-c$ am kleinsten, nach jeder ersten Systole einer Gruppe ist das nämliche Intervall schon viel länger, und es wächst bei jeder Systole, bis schließlich kein c auf a folgt; dadurch entsteht wioder eine Pause, und das Spiel wiederholt sich.

Diese Erklärung dieser Verhältnisse ist diejenige, welche Wenckebach für ähnliche Pulsunregelmäßigkeiten gegeben und auch auf von mir publizierte Fälle mit gutem Erfolge angewendet hat. Die Verlängerung des Intervalls a-c $\left(A_{s}-V_{s}\right)$ wird von ihm einer Storung der Reizleitung von $A$ nach $V$ zugeschrieben. Das Leitungsvermögen des Herzmuskels bedingt nämlich das zeitliche Verhältnis zwischen den Kontraktionen der einzelnen Abteilungen des Herzens. Bei jeder Systole wird diese Eigenschaft des Herzmuskels (mit der Reizbarkeit und der Kontraktilität) aufgehoben. Sie kehrt allmählich in der Diastole zuruck. Ist die Reizleitung stark geschådigt, so wird dieselbe. nach einigen Systolen ungenügend, das Intervall $A_{s}-V_{s}$ wird länger, schlieBlich wird der Reiz nicht mehr geleitet, und es fallt eine $V_{s}$ aus.
(Vergl. Wenckebach l. c. $\$ 20$ bis $\S 30$.) In der dadurch entstandenen Pause hat das Leitungsvermögen längere Zeit zur Erholung. Nach der Pause ist daher das Intervall $A_{s}-\nabla_{s}$ (in dem Venenpulse $a-c$ ) am kürzesten, bald aber ist es durch die Systolen wieder orschöpft, und es tritt wieder oine Pause ein.

Dieser Fall ist geeignet, noch eine andere Ansicht Wenckeb a ch s zu bestätigen. Er hat schon früher ausgeführt, daB regelmäBige Intermission durch Störung der Reizleitung eine Kontraindikation für Digitalis abgebe, weil Digitalis, vielleicht durch Vagusreizung, das Leitungsvermögen negativ zu beeinflussen im stande wäre. Daß hier die Digitalis tatsächlich die Ursache der Störung war, geht ohne weiteres aus der Krankengeschichte hervor. Man könnte sich nun fragen, ob nicht vor dem Auftreten der Allorhythmie schon ein Zeichen gestörter Reizleitung vorhanden war. Als solches wäre vielleicht das anch im regelmäßigen Stadium schon zu lange Intervall $a-c$ anzusehen. Allerdings muß dabei bemerkt werden, daß die Frequenz eine hohe war, daher das Leitungsvermögen nach jeder Systole weniger Zeit zur Erholung hatte, als bei weniger frequenter Herztätigkeit. $\mathrm{Ob}$ Jeitungsstörungen auch die einzige Ursache der Verlängerung dieses Intervalls sind, kann ich nicht entscheiden; aber jedenfalls wird die hier gemachte Erfahrung mich dazu führen, in Fällen, wo man ein zu langes Intervall $A_{s}-V_{s}$ feststellen kann, mit der Darreichung von Digitalis auberst vorsichtig zu sein. 